\title{
Optical phonon features of triclinic montebrasite: Dispersion analysis and non-polar Raman modes
}

\author{
Rafael M. Almeida ${ }^{\mathrm{a}}$, Sonja Höfer ${ }^{\mathrm{b}}$, Thomas G. Mayerhöfer ${ }^{\mathrm{b}}$, Jürgen Popp ${ }^{\mathrm{b}}$, \\ Klaus Krambrock ${ }^{\mathrm{a}}$, Ricardo P.S.M. Lobo ${ }^{\mathrm{c}}$, Anderson Dias ${ }^{\mathrm{d}}$, Roberto L. Moreira ${ }^{\mathrm{a}, *}$ \\ a Departamento de Física, ICEx, Universidade Federal de Minas Gerais, C.P. 702, 30123-970 Belo Horizonte, Minas Gerais, Brazil \\ ${ }^{\mathrm{b}}$ Leibniz-Institut für Photonische Technologien e.V., Albert-Einstein Straße 9, D-07745 Jena, Germany \\ ' Laboratoire de Physique et d'Étude des Matériaux, ESPCI, CNRS, UPMC, 10 rue Vauquelin, F-75231 Paris Cedex 5, France \\ d Departamento de Química, ICEB, Universidade Federal de Ouro Preto, 35400-000 Ouro Preto, Minas Gerais, Brazil
}

\section{A R T I C L E I N F O}

\section{Article history:}

Received 17 September 2014

Received in revised form 16 December 2014

Accepted 16 February 2015

Available online 18 February 2015

\section{Keywords:}

Triclinic crystals

Infrared spectroscopy

Raman spectroscopy

Dispersion analysis

Dielectric response

\begin{abstract}
A B S T R A C T
Polarized infrared and Raman spectra of triclinic $\mathrm{LiAl}\left(\mathrm{PO}_{4}\right)(\mathrm{OH})$ [montebrasite] single crystal were recorded for appropriate optical configurations. Dispersion analysis was applied on the infrared reflectivity spectra taken at low incidence angle $\left(11^{\circ}\right)$ to determine the oscillator parameters and the dipole directions of the polar phonons. In particular, all the 27 polar phonons, predicted by group theory for triclinic P $\overline{1}$ structure, were determined. The obtained dielectric tensor parameters have been checked by comparison between predicted and measured infrared spectra at higher incidence angle $\left(34^{\circ}\right)$. The azimuth and co-elevation angles obtained from the dispersion analysis showed that the response of several polar phonons is close to that of an orthorhombic system. Polarized Raman spectra obtained in several scattering geometries allowed us to obtain well-defined 24 non-polar modes, also in perfect agreement with group theory. The selection rule between Raman and infrared phonons was respected, confirming the centrosymmetric structure and ruling out any relevant influence of defects. The relatively narrow phonon bands are compatible with a highly ordered structure with fully occupied atomic sites.
\end{abstract}

(c) 2015 Elsevier B.V. All rights reserved.

\section{Introduction}

The optical properties of insulator crystals in the infrared spectral region are essentially determined by the characteristics of their vibrational (i.e., phononic) excitations. The optical phonons are usually obtained from inelastic Raman scattering or infrared spectroscopy (transmission or reflection), whose activities depend on several particular features of the system/measuring configuration, mainly the crystal symmetry, sample orientation, light polarization and propagation directions. The knowledge of the phonon features of a material brings information about crystal structure and quality, as well as its optical and dielectric properties, useful for designing technological applications.

The determination of the polar phonon parameters (i.e., frequency, strength and damping constants) of a system from its infrared reflectivity spectra is called dispersion analysis (DA). It was firstly developed by Czerny for cubic crystals [1], and later extended to lower symmetry systems by using polarized infrared

\footnotetext{
* Corresponding author. Tel.: +55 313409 5624; fax: +55 3134095600 . E-mail address: bmoreira@fisica.ufmg.br (R.L. Moreira).
}

spectra measured along their crystallographic axes [2,3], taking benefit of the fact that those axes are the principal ones of the dielectric tensor function for all systems with orthogonal or higher symmetries (i.e., cubic, tetragonal, orthorhombic, rhombohedral or hexagonal structures). Once the polar phonon characteristics of the system are known, the dielectric tensor function can be derived in the spectral region of interest [4,5]. While DA methodology is very well established for higher symmetry systems, the extension for monoclinic and triclinic crystals is not straightforward, since for these non-orthogonal systems the dielectric function always has non-diagonal terms. In terms of the polar phonons, this implies that their dipoles (or transition moments) are no longer parallel to the crystallographic axes, and are indeed at individually defined directions in the plane of non-orthogonal axes (monoclinic crystals) or in space (triclinic ones). Because of the increased difficulties of working with low-symmetry systems, only a bit more than a dozen of works on monoclinic [6-22] and three on triclinic [23-26] single-crystalline samples were reported so far using DA, at the best of our knowledge. Very recently, some of us (S. H. \& T.M.) introduced a new DA methodology for investigating triclinic crystals. This method has been successfully applied to blue vitriol and potassium dichromate, revealing that the optical 
properties of the first one look like those of orthorhombic crystals [25], while the second one deviates strongly from this lower symmetry [26]. In the present work, we use DA for investigating a montebrasite crystal $\left[\mathrm{LiAl}\left(\mathrm{PO}_{4}\right)(\mathrm{OH}]\right]$ with triclinic structure, aiming to bring information about the optical and structural features of this system.

Montebrasite is the high hydroxyl end member of the amblygonite-montebrasite system, $\operatorname{LiAl}\left(\mathrm{PO}_{4}\right)(\mathrm{F}, \mathrm{OH})$. In this system, the amount of fluorine or hydroxyl anions can vary from 0 to 1 maintaining the crystal structure, which was shown to belong to the centrosymmetric $\mathrm{P} \overline{1}$ space group [27]. Montebrasite is a common mineral in granitic pegmatite of lithium, being an important source of this alkali metal for battery applications. It also has economic importance as a source of phosphate for fertilizers in agriculture [28], though amblygonitemontebrasite minerals are undesirable contaminants in spodumene in the refractory industry [29]. Crystals with good quality for the gemstone market are relatively rare, and practically all come from Brazilian pegmatites, which favor montebrasite over amblygonite [30]. Indeed, petrological studies revealed that high fluorine containing amblygonite is rare in granitic pegmatites, being present mainly in topaz-bearing granites [31]. In addition to the above cited economic and petrological interest, amblygonitemontebrasite system attracts scientific interest due to the possibility of studying their phase diagram (complete solid solution between $\mathrm{F}$ and $\mathrm{OH}$ end members) [28-33]. In the present work, a very good gemstone quality montebrasite was chosen for investigation of the intrinsic optical vibrational properties of this triclinic material, by combining dispersion analysis of the infrared reflectance spectra with polarized Raman scattering spectra.

\section{Experimental}

A natural single-crystal specimen of montebrasite from granitic pegmatite of the Telírio mine (Linópolis, Divino Laranjeiras, Minas Gerais, Brazil) was chosen for this work. This mine provides the best montebrasite gemstones of the world. The sample studied here has been characterized for its chemical formula, $\mathrm{F} / \mathrm{OH}$ contents and metal impurities, and the results were published in a previous work, where the sample received the code AAM [28]. Our sample presented less than $1 \%$ of metal impurities (Na, Ti, Fe, $\mathrm{Nb}, \mathrm{V})$, and less than $3 \%$ of fluorine ( $97 \%$ of $\mathrm{OH})$, allowing us to write its formula in good approximation as $\operatorname{LiAl}\left(\mathrm{PO}_{4}\right)(\mathrm{OH})$. The sample has been cut from a larger gemstone of excellent optical quality that was highly transparent (slightly yellowish), in a piece with $2.5 \mathrm{~mm} \times 2.0 \mathrm{~mm} \times 4.5 \mathrm{~mm}$, in the form of a parallelepiped. For cutting the sample we used the orientation of perfect cleavage planes normal to [100] axis and good cleavage normal to [110]

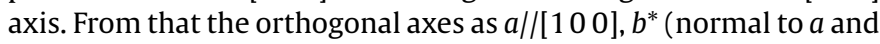
close to $b$, once the [110] cleavage direction is known), and $c^{*}$, parallel to $a \times b^{*}$ were defined [28]. Three corner-sharing faces were polished to optical grade to mimic the three adjacent faces of a cube.

The triclinic montebrasite structure has been determined as belonging to the centrosymmetric $\mathrm{P} \overline{1}$ space group, with unit cell parameters $a=5.18 \AA, b=7.15 \AA$ and $c=5.04 \AA$, with $\alpha=112.07^{\circ}$, $\beta=97.48^{\circ}$ and $\gamma=67.53^{\circ}$ [27]. This structure is characterized by distorted $\mathrm{AlO}_{4}(\mathrm{OH})_{2}$ octahedra interconnected along the [110] direction by common hydroxyl groups. These octahedra are laterally interconnected by nearly perfect $\mathrm{PO}_{4}$ tetrahedra, while the $\mathrm{Li}$ ions occupy the cavities left by the octahedral chains and $\mathrm{PO}_{4}$ tetrahedra $[27,28]$. The unit cell has two formula units $(Z=2)$. In this description, there are two non-equivalent aluminum positions with special $C_{i}$ symmetry, while all other ions occupy general positions with $C_{1}$ symmetry. All Wyckoff positions are fully occupied. This structure differs slightly from that proposed earlier by Simonov and Bjelov [34], who proposed two half-occupied non-equivalent lithium ions in general positions ( $C_{1}$ symmetry). We will return to this difference after presenting our spectroscopic results. A quite useful alternative description of montebrasite structure was proposed by Groat et al. [31,33]. These authors proposed a hypothetical pseudo-monoclinic structure for describing montebrasite-amblygonites, owing to the chemical and structural similarity between these materials and several important minerals (e.g., kieserite $\mathrm{MgSO}_{4} \mathrm{H}_{2} \mathrm{O}$, and titanite $\mathrm{CaTiSiO}_{4} \mathrm{O}$ ). The unit cell for montebrasite would have $a=6.713 \AA$, $b=7.708 \AA$ and $c=7.0194 \AA$, with $\alpha=91.31^{\circ}, \beta=117.93^{\circ}$ and $\gamma=91.77^{\circ}$ and $Z=4$ [33]. In this unit cell the octahedral chains are along the pseudomonoclinic c-axis. Again, in this description, two non-equivalent half-occupied lithium ions were assumed. However, by revisiting the problem later [31], Groat et al. proposed that the occupancies of lithium in these two sites could be different from 0.5 and vary with temperature. As we have already indicated, our spectroscopic results allow us to discuss on these structural features.

Polarized infrared measurements of the montebrasite crystal were performed by using two different Fourier transform spectrometers (FTIR). The mid-infrared spectra $\left(550-4000 \mathrm{~cm}^{-1}\right)$ were collected under purge on a Nexus 470 (Thermo-Nicolet) with a $\mathrm{SiC}$ source, a $\mathrm{KBr}: \mathrm{Ge}$ beamsplitter, an MCT detector and a grid polarizer onto a $\mathrm{ZnSe}$ substrate as standard accessories for this configuration. The far-infrared spectra $\left(50-700 \mathrm{~cm}^{-1}\right)$ were collected under vacuum (1 Torr) on a DA-8 (BOMEM) spectrometer equipped with a SiC source, a mylar hypersplitter ${ }^{\circledR}$, a Si-bolometer detector and a wire grid polarizer onto a PE substrate. The reflectivity spectra in both spectral regions were obtained either, for low-incidence angle $\left(11^{\circ}\right)$ or high-incidence angle $\left(34^{\circ}\right)$, with two different reflection accessories. The spectra were recorded by averaging at least 128 scans for each polarization, with the spectral resolution better than $4 \mathrm{~cm}^{-1}$. Gold mirrors were used for obtaining the reference spectra. The infrared reflectivity spectra recorded in mid- and far-infrared regions matched well in the superposition region.

Polarized Raman spectra were collected at room temperature on the three sample surfaces, in parallel and crossed configurations, using a Horiba/Jobin-Yvon LABRAM-HR spectrometer, equipped with a CCD detector and a confocal Olympus microscope $(100 \times$ objective). The measurements were carried out in back-scattering geometry, for varying angles between sample edges and light-beam polarizations, using the $633 \mathrm{~nm}$ line of He-Ne laser $(6 \mathrm{~mW})$ as excitation source. Appropriate interference and edge filters, half-wave plate and polarizer were used. The spectra were obtained between 50 and $3800 \mathrm{~cm}^{-1}$, with spectral resolution of about $1 \mathrm{~cm}^{-1}$, by averaging 10 accumulations of $30 \mathrm{~s}$.

\section{Theoretical background}

Dispersion analysis (DA) of the FTIR reflectivity spectra of montebrasite was performed by using the method described in Refs. [25] and [26], and summarized in the sequence. In the DrudeLorentz model the dielectric tensor function $\varepsilon_{x, y, z}(\tilde{v})$ is the sum of the damped harmonic Lorentz oscillators, which describe the fingerprints occurring in an IR spectrum due to vibrations, plus a dielectric background tensor $\left[\varepsilon_{\infty}\right]$, which is a (constant) offset summarizing all contributions from the UV and visible part of the spectrum.

For a dielectric non-magnetic and optically inactive triclinic crystal the dielectric tensor function has the following form: 


$$
\begin{aligned}
& \left.\varepsilon_{x, y, z}(\tilde{v})=\left[\begin{array}{lll}
\varepsilon_{\infty, x x} & \varepsilon_{\infty, x y} & \varepsilon_{\infty, x z} \\
\varepsilon_{\infty, y x} & \varepsilon_{\infty, y y} & \varepsilon_{\infty, y z} \\
\varepsilon_{\infty, z x} & \varepsilon_{\infty, z y} & \varepsilon_{\infty, z z}
\end{array}\right]+\sum_{i=1}^{N} \frac{S_{i}^{2}}{\left(\tilde{v}_{i}\right.}-\tilde{v}^{2}\right)-i \gamma_{i} \tilde{v} \\
& \quad \times\left(\begin{array}{ccc}
\sin ^{2} \Theta_{i} \cos ^{2} \Phi_{i} & \sin ^{2} \Theta_{i} \sin \Phi_{i} \cos \Phi_{i} & \sin \Theta_{i} \cos \Theta_{i} \cos \Phi_{i} \\
\sin ^{2} \Theta_{i} \sin \Phi_{i} \cos \Phi_{i} & \sin ^{2} \Theta_{i} \sin ^{2} \Phi_{i} & \sin \Theta_{i} \cos \Theta_{i} \sin \Phi_{i} \\
\sin \Theta_{i} \cos \Theta_{i} \cos \Phi_{i} & \sin \Theta_{i} \cos \Theta_{i} \sin \Phi_{i} & \cos ^{2} \Theta_{i}
\end{array}\right)
\end{aligned}
$$

In Eq. (1) the oscillator parameters are the resonance wavenumber, $\tilde{v}_{i}$, the oscillator strength, $S_{i}$, and the damping constant, $\gamma_{i}$. The two angles $\Phi_{i}$ and $\theta_{i}$ describe the orientation of the transition moment of the $i$ th oscillator relative to a right-handed coordinate system $(x$, $y, z)$ that is fixed within the crystal. The definition of $\Phi$ and $\theta$ is the same as that used in spherical coordinates, i.e., co-elevation and azimuth angles. The index $i$ denotes the number of the oscillator, so the sum runs over all oscillators present in the spectral part of interest. As there are no symmetry elements in a triclinic crystal (except for a possible inversion center), there is no predominant orientation for the transition moments within the crystal. As a consequence, the dielectric axes vary with frequency $\left(\Phi_{i}\right.$ and $\theta_{i}$ depend on $\tilde{v}$ ), and the real and imaginary parts of the dielectric tensor have generally different main axes.

The measurement scheme of triclinic crystals developed in [25] requires three orthogonal and optically polished faces carved out of the crystal. For convenience, the cut crystal is depicted as a cube. Fig. 1 depicts the employed coordinate systems. To record the spectra, the cube is rotated into six different orientations, which are shown in Fig. 2. For each one of the three orthogonal faces, four spectra with different polarization directions of the incoming light are recorded, so the number of measured spectra sums up to 12 , as shown in Fig. 3.

To describe the rotation of the sample within the spectrometer and to determine the dielectric tensor function $\varepsilon_{x, y, z}(\tilde{v})$ in laboratory coordinates $(X, Y, Z)$, the tensor function is multiplied by a rotation matrix $\mathbf{A}(\varphi, \theta, \psi)$. The rotation matrix is a function of the Euler angles $\phi, \theta$, and $\psi$ which specify the rotation of the crystal sample in the spectrometer.

$$
\begin{aligned}
& \varepsilon_{X, Y, Z}=\boldsymbol{A}(\varphi, \theta, \psi) \times \varepsilon_{x, y, z} \times \boldsymbol{A}^{-1}(\varphi, \theta, \psi) \\
& \boldsymbol{A}(\varphi, \theta, \psi)=\boldsymbol{A}(\varphi) \times \boldsymbol{A}(\theta) \times \boldsymbol{A}(\psi)
\end{aligned}
$$

$\boldsymbol{A}(\phi)$ is a rotation matrix which describes a rotation around the (fixed) $Z$-axis, $\boldsymbol{A}(\theta)$ a rotation around the (fixed) $Y$-axis and $\boldsymbol{A}(\psi)$ again a rotation around the $Z$-axis.

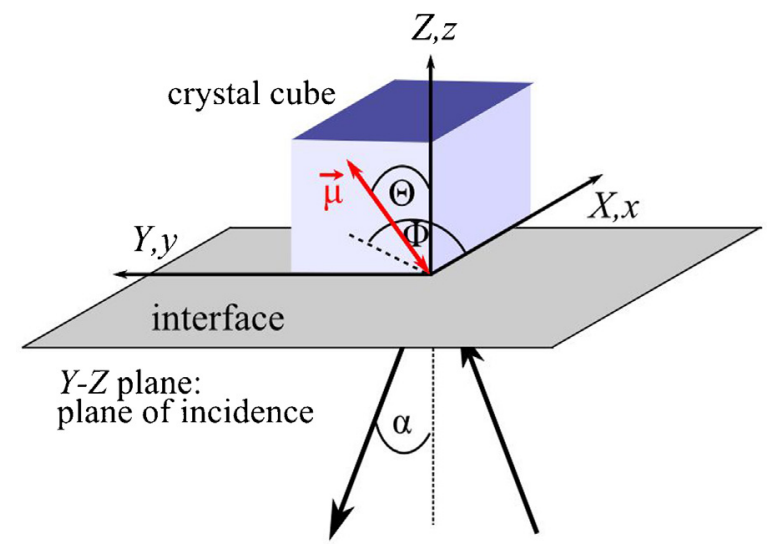

Fig. 1. Applied coordinate systems and geometry for reflection measurements. (X,Y, $Z$ ) are the laboratory coordinates, the plane of incidence is given by the $Y-Z$ plane, the interface between crystal sample and vacuum is parallel to the $X-Y$ plane. $(x, y, z)$ are the crystal coordinates fixed within the crystal, corresponding to cell parameters $\left(b^{*}, a,-c^{*}\right)$. The incidence angles were $11^{\circ}$ and $34^{\circ}$.
To yield expressions for the reflectivity of the calculated spectra the first order Maxwell equation:

$$
\frac{\partial}{\partial Z} \boldsymbol{\Psi}=i k_{0} \Delta \boldsymbol{\Psi}
$$

is solved employing the dielectric tensor function. Eq. (3) describes the dependence of the field vector $\boldsymbol{\Psi}=\left(E_{X}, H_{Y}, E_{Y}, H_{X}\right)^{T}$ in $Z$-direction. For the wave vector $\boldsymbol{k}=k_{0}(0, \sin \alpha, \cos \alpha)^{T}$ we assume a plane wave with no component in $X$-direction, as the wave is travelling in the $Z-Y$ plane. The $\Delta$-matrix is given by [25]:

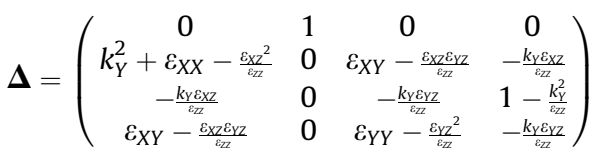

where in the $\varepsilon_{i j}$ are the components of the dielectric tensor function. The solution of the eigenvalue problem of Eq. (4) is given by the determinant equation $\operatorname{Det}(\Delta-\lambda \times I)=0$, with $\lambda$ being the four eigenvalues of the determinant equation and $\boldsymbol{I}$ the identity matrix. Two of the four eigenvalues are either real or have a positive imaginary part and describe the forward travelling waves; the two other describe the backward travelling waves. From the corresponding eigenvectors $\boldsymbol{\Psi}$ the dynamical matrix $\boldsymbol{D}_{\boldsymbol{\Psi}, \text { crystal }}$ is generated:

$D_{\Psi, \text { crsytal }}=\left(\begin{array}{llll}\Psi_{I, 1} & 0 & \Psi_{I I, 1} & 0 \\ \Psi_{I, 2} & 0 & \Psi_{I I, 2} & 0 \\ \Psi_{I, 3} & 0 & \Psi_{I I, 3} & 0 \\ \Psi_{I, 4} & 0 & \Psi_{I I, 4} & 0\end{array}\right)$

Due to the assumption of a semi-infinite medium the second and fourth column, which describe the backward travelling waves of the dynamical matrix can be set to zero. The indices $i j$ of $\boldsymbol{\Psi}_{\boldsymbol{i j}}$ describe the eigenvector corresponding to the eigenvalue $\lambda_{i}$ and the components of $\boldsymbol{\Psi}=\left(E_{X}, H_{Y}, E_{Y}, H_{X}\right)^{T}$, respectively.

The dynamical matrix $\boldsymbol{D}_{\boldsymbol{\Psi}}$, crystal of the crystal and the inverse dynamical matrix of the incidence medium (air or vacuum), $\boldsymbol{D}_{\boldsymbol{\Psi} \text {,inc, }}$ link the amplitudes of the incident $\left(A_{s}, A_{p}\right)$, reflected $\left(B_{s}, B_{p}\right)$ and transmitted $\left(C_{s}, C_{p}\right)$ waves [35].

$$
\begin{aligned}
\boldsymbol{M} & =\boldsymbol{D}_{\boldsymbol{\Psi}, \text { inc }}^{-1} \times \boldsymbol{D}_{\boldsymbol{\Psi}, \text { crystal }} \text { and }\left(\begin{array}{c}
A_{\mathrm{s}} \\
B_{\mathrm{s}} \\
A_{\mathrm{p}} \\
B_{\mathrm{p}}
\end{array}\right) \\
& =\left(\begin{array}{llll}
M_{11} & M_{12} & M_{13} & M_{14} \\
M_{21} & M_{22} & M_{23} & M_{24} \\
M_{31} & M_{32} & M_{33} & M_{34} \\
M_{41} & M_{42} & M_{43} & M_{44}
\end{array}\right) \cdot\left(\begin{array}{c}
C_{\mathrm{s}} \\
0 \\
C_{\mathrm{p}} \\
0
\end{array}\right)
\end{aligned}
$$

With Eq. (6) the measured quantities $R_{\mathrm{s}}$ and $R_{\mathrm{p}}$ can explicitly be calculated with the reflection coefficients $r_{i j}$. The index $i$ describes the polarization state of the polarizer and $j$ the polarization state of the analyzer. As no analyzer was used, the reflectivities $R_{\mathrm{S}}$ and $R_{\mathrm{p}}$ are $R_{\mathrm{s}}=R_{\mathrm{ss}}+R_{\mathrm{sp}}=r_{\mathrm{ss}} r_{\mathrm{ss}}{ }^{*}+r_{\mathrm{sp}} r_{\mathrm{sp}}{ }^{*}$ and $R_{\mathrm{p}}=R_{\mathrm{ps}}+R_{\mathrm{pp}}=r_{\mathrm{pp}} r_{\mathrm{pp}}{ }^{*}+r_{\mathrm{ps}} r_{\mathrm{ps}}{ }^{*}$ and the equations for $r_{i j}$ as given below $\left(r_{i j}{ }^{*}\right.$ is the complex conjugate of $r_{i j}$ ).

$$
\begin{aligned}
& r_{\mathrm{ss}}=\left(\frac{B_{\mathrm{s}}}{A_{\mathrm{s}}}\right)=\frac{M_{21} M_{33}-M_{23} M_{31}}{M_{11} M_{33}-M_{13} M_{31}} \\
& r_{\mathrm{sp}}=\left(\frac{B_{\mathrm{p}}}{A_{\mathrm{s}}}\right)=\frac{M_{41} M_{33}-M_{43} M_{31}}{M_{11} M_{33}-M_{13} M_{31}} \\
& r_{\mathrm{ps}}=\left(\frac{B_{\mathrm{s}}}{A_{\mathrm{p}}}\right)=\frac{M_{11} M_{23}-M_{21} M_{13}}{M_{11} M_{33}-M_{13} M_{31}} \\
& r_{\mathrm{pp}}=\left(\frac{B_{\mathrm{p}}}{A_{\mathrm{p}}}\right)=\frac{M_{11} M_{43}-M_{41} M_{13}}{M_{11} M_{33}-M_{13} M_{31}}
\end{aligned}
$$


$X, Y, Z:$ Laboratory coordinate system, $\quad x, y, z$ : crystal coordinate system, fixed in crystal

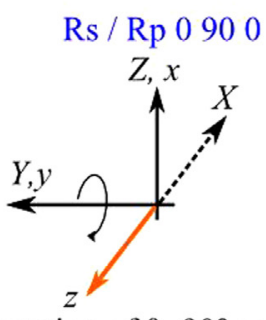

Rotation of $\theta=90^{\circ}$ around $Y$<smiles>[CH]=C</smiles>

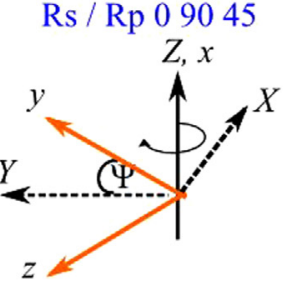

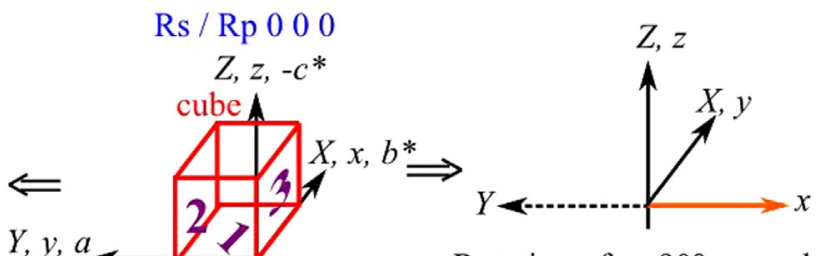

Initial Orientation

Rotation of $\varphi=90^{\circ}$ around $Z$

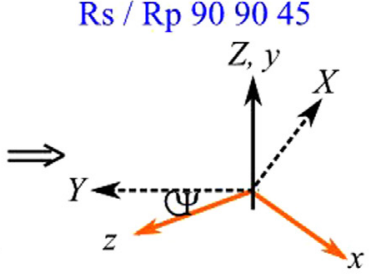

Rotation of $\theta=90^{\circ}$ around $Y$ +rotation of $\Psi=45^{\circ}$ around $Z$

$\Downarrow$

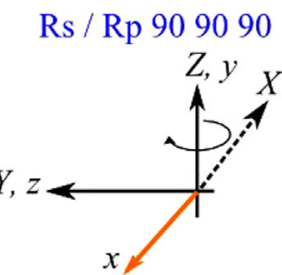

Rotation of further $\Psi=45^{\circ}$ around $Z$

measuring face 3

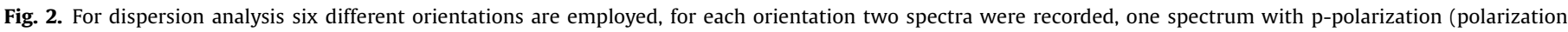

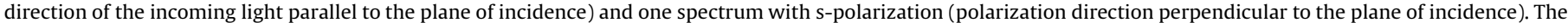

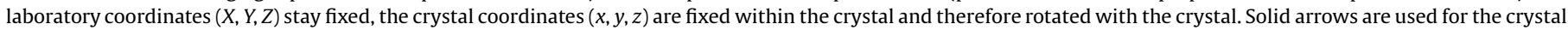

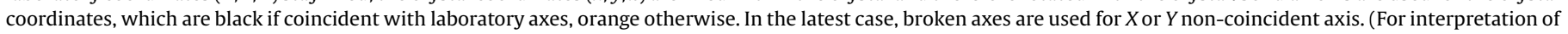
the references to color in this figure legend, the reader is referred to the web version of this article.)

\section{Results and discussion}

\subsection{Dispersion analysis of montebrasite}

Montebrasite belongs to the space group $C_{i}^{1}$ with all atoms occupying general $C_{1}$ positions except for the $\mathrm{Al}$ ions, which occupy two non-equivalent special positions with $C_{i}$ symmetry [27]. According to [36] each of the $8 C_{1}$ positions contributes with $3 \mathrm{~A}_{\mathrm{g}}+3 \mathrm{~A}_{\mathrm{u}}$ normal modes and each of the two special positions contributes with $3 \mathrm{~A}_{\mathrm{u}}$ modes. These normal modes sum up to

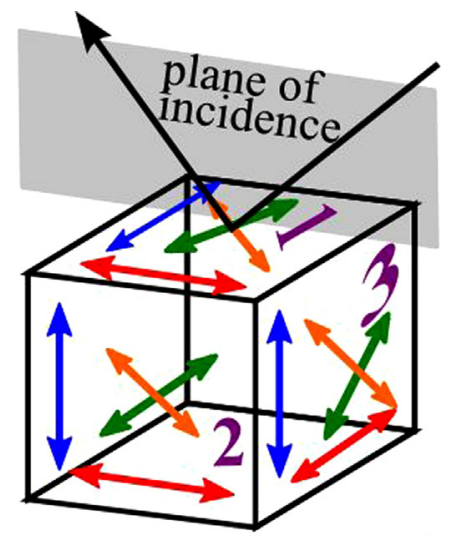

$\begin{array}{lll}\text { Face 1: } & \text { Face 2: } & \text { Face } 3: \\ \mathrm{R}_{\mathrm{s}} \text { 00 } & \mathrm{R}_{\mathrm{s}} \text { 090 } & \mathrm{R}_{\mathrm{p}} 909090 \\ \mathrm{R}_{\mathrm{p} 000} & \mathrm{R}_{\mathrm{p}} \text { 000 } & \mathrm{R}_{\mathrm{s}} 909090 \\ \mathrm{R}_{\mathrm{p} 4500} & \mathrm{R}_{\mathrm{p}} \text { 09045 } & \mathrm{R}_{\mathrm{s}} 909045 \\ \mathrm{R}_{\mathrm{S}} 4500 & \mathrm{R}_{\mathrm{S}} \text { 09045 } & \mathrm{R}_{\mathrm{p}} 909045\end{array}$

Fig. 3. Illustration of how the different spectra and polarization directions created by the six rotations are distributed on the crystal cube. The numbers 1,2 , 3 numerate the cube faces.
$24 A_{g}+30 A_{u}$ modes, of which the $A_{g}$ modes are Raman active and the $A_{u}$ modes are IR active. Among the $30 A_{u}$ modes, $3 A_{u}$ modes have to be subtracted as acoustic modes (translation of the crystal), so there remain 27 IR modes to be observed. By analyzing altogether the 12 low-incidence angle FTIR spectra of montebrasite with our optimization routine we found 26 oscillators below $1500 \mathrm{~cm}^{-1}$. The fitted oscillator parameters are listed in Table 1. The parameters shown in this table have usual classical meaning (they are intrinsic to the crystal and independent on the measuring plane), but the azimuth and co-elevation angles are referred to a particular measuring configuration. In the present case, the crystal coordinates $(x, y, z)$ fixed in the crystal correspond to the cut $\left(b^{*}, \mathrm{a}\right.$, $\left.-c^{*}\right)$ of our sample. The correspondence between the reflectivity spectra and crystal orientation (measuring planes and directions) is shown in Table 2. At around $3400-3350 \mathrm{~cm}^{-1}$ most spectra exhibit the $\mathrm{O}-\mathrm{H}$ stretching vibrations; however we were not able to find suitable Lorentz oscillators to fit this vibration.

The spectral region of the $\mathrm{O}-\mathrm{H}$ stretching vibration is separately depicted in Fig. 4. The vibration does not occur in the spectra $R_{\mathrm{p}} 909090$ (electric field $\left.E / / c^{*}\right), R_{\mathrm{s}} 909090\left(E / / b^{*}\right), R_{\mathrm{p}} 9090$ $45\left(E / / b^{*}+c^{*}\right)$ and $R_{\mathrm{s}} 909045\left(E / / b^{*}-c^{*}\right)$, which means that the transition moments creating this vibration is oriented perpendicularly to face $3\left(b^{*} c^{*}\right)$, i.e., to the cleavage plane of montebrasite. This result agrees with previous structural studies of this crystal, which show that the $\mathrm{OH}$ bond is oriented approximately along its crystallographic $a$-axis $[27,28]$. Consequently, the $\mathrm{OH}$ stretching vibration should have maximal reflectivity in spectra $R_{\mathrm{p}} 000 / 090$ $0(E / / a)$, as it is confirmed by the two upper spectra on the left panel of Fig. 4. The $R_{\mathrm{S}} 000\left(E / / b^{*}\right)$ and $R_{\mathrm{s}} 0900\left(E / / c^{*}\right)$, respectively on planes $1\left(a b^{*}\right)$ and $2\left(a c^{*}\right)$, show weak intensity for this vibration, indicating smaller (but non-zero) components of the corresponding transition moment along these directions. This can explain the slightly higher intensities of this vibration for the $R_{\mathrm{p}} 4500$ (E// $\left.a+b^{*}\right)$ over $R_{\mathrm{s}} 4500\left(E / / a-b^{*}\right)$ on plane $1\left(a b^{*}\right)$, and $R_{\mathrm{p}} 09045(E / /$ $\left.a+c^{*}\right)$ over $R_{\mathrm{s}} 09045\left(E / / a-c^{*}\right)$ on plane $2\left(a c^{*}\right)$. As a whole, concerning the amplitude of the reflectivity, the spectral 
Table 1

Dispersion parameters for montebrasite: resonance frequency $\tilde{v}_{i}$, strength $S_{i}$, damping $\gamma_{i}$, all three in $\mathrm{cm}^{-1}$, and angles $\Phi_{i}$ and ${ }_{i}$ in degree. The dielectric background tensor $\varepsilon_{\infty}$ is without units. The oscillators are given with one decimal and two decimals in case of the dielectric background tensor as they were calculated by the optimization, although the digits do not reflect the accuracy of the parameters.

$S_{i} \quad \gamma_{i} \quad \Phi_{i}$

$\tilde{v}_{i}$

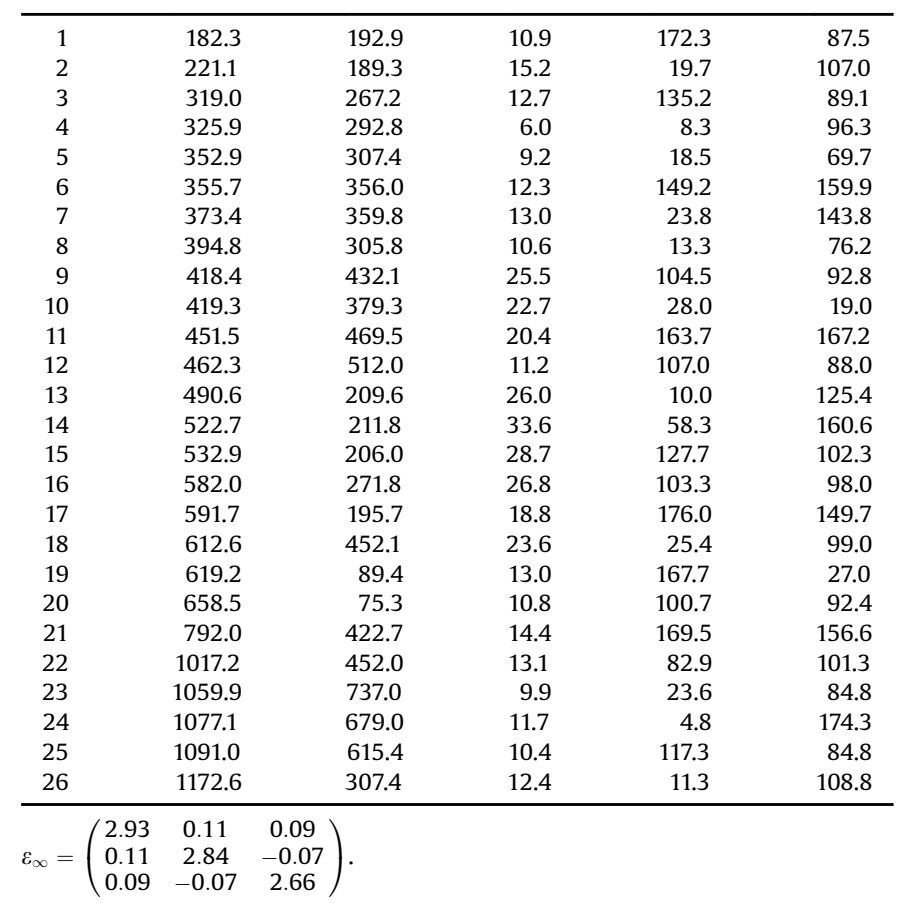

characteristics of the $\mathrm{OH}$ stretching vibration in the different used polarized configurations are quite consistent. However, a very intriguing frequency dispersion occurred for the different measured faces. Indeed, the characteristic frequency of the vibration presented an almost $25 \mathrm{~cm}^{-1}$ upshift on all spectra taken on face 2 $\left(a c^{*}\right)$ compared to face $1\left(a b^{*}\right)$. This upshift cannot be described by our theoretical model based upon independent Lorentz oscillators. For this reason, our fitting curves on Fig. 4 are all at the same (average) frequency position and cannot describe the observed dispersion. The mechanism underlying such effect is unknown at the light of the experimental data available so far and is beyond the scope of the present work. It deserves further investigation and should be addressed in future works.

The $\mathrm{PO}_{4}^{3-}$ tetrahedra contribute with $5 \times\left(3 \mathrm{~A}_{\mathrm{g}}+3 \mathrm{~A}_{\mathrm{u}}\right)=15 \mathrm{~A}_{\mathrm{g}}$ Raman active $+15 A_{u}$ IR active modes. The IR active modes consist of 6 librational modes and 9 internal modes, $3 \times v_{3}\left(A_{u}\right), 1 \times v_{1}\left(A_{u}\right)$, $3 \times v_{4}\left(A_{u}\right)$ and $2 \times v_{2}\left(A_{u}\right)$ [36]. With the help of [30,32,37-40] a tentative band assignment can be made for the calculated oscillators, although band assignment is complicated by resonance of dipole-dipole interactions between $\mathrm{PO}_{4}^{3-}$ groups, which can lead to additional band splitting $[41,42]$, and also because the low frequency external modes are linear combinations of motions involving several ions. Oscillator 26 around $1173 \mathrm{~cm}^{-1}$ is likely the $\mathrm{OH}$-bending mode, as often observed in related materials [40]. Oscillators 22-25 most probably contain the $3 \times v_{3}$ and the $v_{1}$ vibration of the phosphate tetrahedra. Crystal structure analysis revealed only little distorted $\mathrm{PO}_{4}^{3-}$ tetrahedra [27], since oscillators 23-25 are roughly orthogonal within calculation errors (Table 3 ), but oscillator 22 does not fit into the orthogonality. Oscillator 23 has maximum intensity in spectra $R_{\mathrm{p}} 909090, R_{\mathrm{s}} 0900, R_{\mathrm{s}} 9090$ 45 and with a little less intensity in $R_{\mathrm{S}} 09045$. The oscillator is not visible in $R_{\mathrm{p}} 000$, and occurs in the other spectra with very low intensity. From these considerations it follows that the corresponding transition moment must be oriented almost perpendicular to the cube measuring face $1\left(a b^{*}\right)$. This assumption is confirmed by the optimization which found an angle pair with $\Phi=169.5^{\circ}$ and $\theta=156.6^{\circ}$. The spectra show that the vibration at $792 \mathrm{~cm}^{-1}$, corresponding to the oscillator 21 , is a vibration of a linear or two dimensional compound as the reflection intensity strongly depends on the polarization direction of the incoming light. This oscillator is also related to the presence of hydroxyl groups [32], and is probably linked to $\mathrm{P}-\mathrm{OH}$ stretching and $\mathrm{P}-\mathrm{O}-$ $\mathrm{H}$ torsional motions [40]. Oscillators $1+2$ are most probably librational modes of the crystal compounds, as librational modes usually are characterized by low wavenumbers. The remaining oscillators 3-20 accounts for the $3 \times v_{4}$ (asymmetric bending), the $2 \times v_{2}$ (symmetric bending) of the phosphate tetrahedra, the 6 librational modes, and the external lattice modes.

The 12 measured spectra from the cube-shaped montebrasite single crystal are depicted in Fig. 5 together with the corresponding best fitting spectra. Fig. 5 (right) shows the spectra with polarization direction perpendicular to the plane of incidence; Fig. 5 (left) shows the spectra with polarization direction parallel to the plane of incidence. As the angle of incidence was $11^{\circ}$, the p-polarization is not exactly along the cube edges or face diagonals. Diverse test simulations showed that the effect of the deviation of $11^{\circ}$ from normal incidence on the optimization of spectra of triclinic crystals is not measureable due to a large error for the fitted angles [26]. As a whole, the comparison between measured and calculated spectra shows a satisfyingly agreement for the investigated spectral ranges.

For the fittings the number of oscillators was chosen according to the expected number of oscillators, as in the case of montebrasite easily 27 or more additional oscillators can be employed by the optimization if it is based only on the number of

Table 2

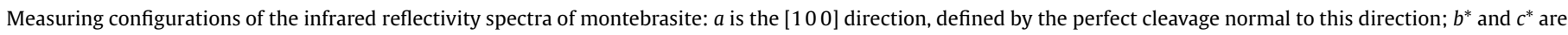

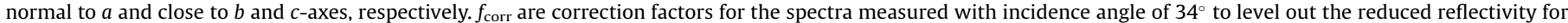
higher incidence angles.

\begin{tabular}{|c|c|c|c|c|c|c|}
\hline Spectrum & $R_{\mathrm{p}} 000$ & $R_{\mathrm{p}} 0900$ & $R_{\mathrm{p}} 909090$ & $R_{\mathrm{p}} 4500$ & $R_{\mathrm{p}} 09045$ & $R_{\mathrm{p}} 909045$ \\
\hline Face & 1 & 2 & 3 & 1 & 2 & 3 \\
\hline Plane & $a b^{*}$ & $\mathrm{ac}^{*}$ & $b^{*} c^{*}$ & $a b^{*}$ & $\mathrm{ac}^{*}$ & $b^{*} c^{*}$ \\
\hline Direction & $\mathrm{a}$ & $\mathrm{a}$ & $c^{*}$ & $a+b^{*}$ & $a+c^{*}$ & $c^{*}+b^{*}$ \\
\hline$f_{\text {corr }}$ & 0.63 & 0.86 & 0.81 & 0.57 & 0.77 & 0.79 \\
\hline Spectrum & $R_{\mathrm{S}} 000$ & $R_{\mathrm{S}} 0900$ & $R_{\mathrm{s}} 909090$ & $R_{\mathrm{S}} 4500$ & $R_{\mathrm{s}} 09045$ & $R_{\mathrm{s}} 909045$ \\
\hline Face & 1 & 2 & 3 & 1 & 2 & 3 \\
\hline Plane & $a b^{*}$ & $\mathrm{ac}^{*}$ & $b^{*} c^{*}$ & $a b^{*}$ & $\mathrm{ac}^{*}$ & $b^{*} c^{*}$ \\
\hline Direction & $b^{*}$ & $c^{*}$ & $b^{*}$ & $a-b^{*}$ & $a-c^{*}$ & $c^{*}-b^{*}$ \\
\hline$f_{\text {corr }}$ & 0.52 & 0.59 & 0.61 & 0.39 & 0.73 & 0.61 \\
\hline
\end{tabular}



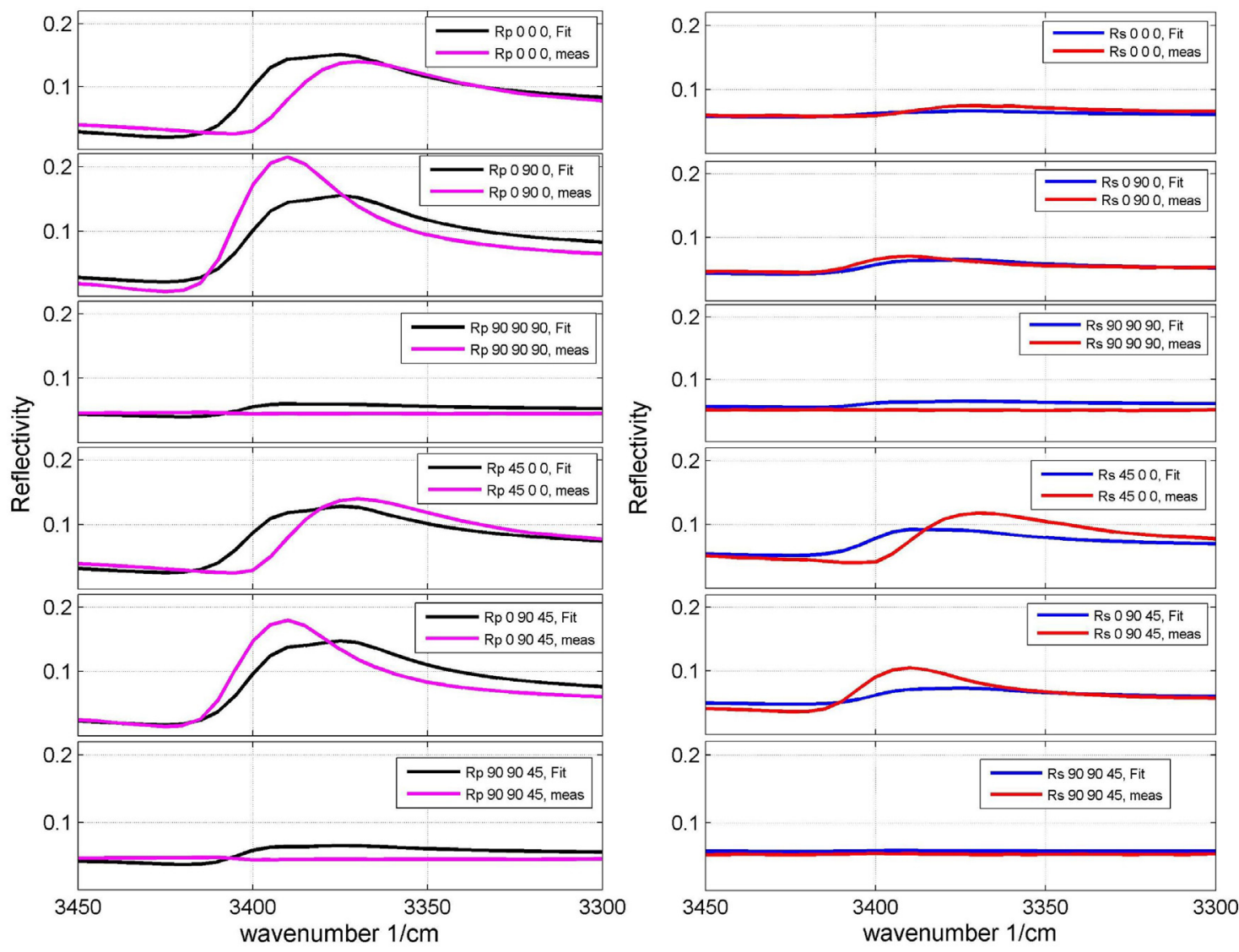

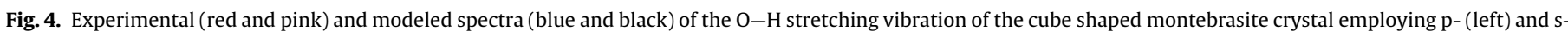

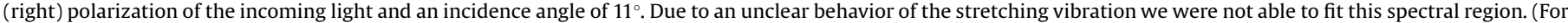
interpretation of the references to color in this figure legend, the reader is referred to the web version of this article.)

bands present in the measured spectral range. Too many oscillators result in particular in the case if many weaker oscillators are spectrally very close as it is the case in $700-100 \mathrm{~cm}^{-1}$.

In Fig. 6 the calculated angle orientation for each of the fitted oscillators is depicted. As is shown in Fig. 1 the calculated angles give the orientation of the transition moment with respect to the normal face of the cube, in which the spectra $R_{\mathrm{S}} / R_{\mathrm{p}} 000$ and $R_{\mathrm{S}} / R_{\mathrm{p}}$ 4500 were recorded, i.e., face $1\left(a b^{*}\right)$. Fig. 6 shows that the transition moments roughly point into three different directions, which means that montebrasite shows an almost orthorhombiclike IR spectral behavior.

\subsection{Verification of the oscillator parameters}

To verify the correctness of the calculated oscillator parameters, the obtained parameters can be employed to predict spectra, e.g., the reflection spectra of a pressed pellet made of crushed sample material, as it was done in $[25,26]$, or another set of spectra with high incidence angle can be recorded. The higher the incidence angle gets, the more the p-polarization of the incoming light excites oscillators of two orthogonal cube faces. The consequence is that for high incidence angles the $R_{\mathrm{p}}$ spectra are mixed spectra of the oscillators of two orthogonal cube faces. The effect is maximal for an incidence angle of $45^{\circ}$. In the present work, as the authors did not want to crush the (gem) crystal, a second set of spectra with an incidence angle of $34^{\circ}$ was measured. To fit the second spectra set the oscillators and dielectric background tensor of Table 1 were employed, the only variable left to optimize was a constant correction factor $f_{\text {corr }}$ for each cube face to level out the reduced reflectivity for higher incidence angles (because of experimental difficulties). The correction factors for the second set of spectra are given in Table 2. Fig. 7 shows the second spectra set optimized with the oscillator parameters gained from the spectra set measured with an incidence angle of $11^{\circ}$. Overall, the spectra for incidence angle $34^{\circ}$ could satisfyingly be reproduced with the oscillator parameters gained from the first spectra set. The most distinct discrepancy between measured and modeled spectra can be found between 1300 and $1100 \mathrm{~cm}^{-1}$ for the $R_{\mathrm{s}} / R_{\mathrm{p}} 4500$ spectra. Since all other modeled spectra resemble the measured ones, we assume that this discrepancy is due to misalignment errors, as especially for triclinic crystals the optimization is quite sensitive to correct orientation of the sample within the spectrometer.

Table 3

Calculated angles between oscillators 22-26.

\begin{tabular}{|c|c|c|c|c|c|c|c|}
\hline Oscillators & Angle $^{\circ}$ & Oscillators & Angle $^{\circ}$ & Oscillators & Angle $^{\circ}$ & Oscillators & Angle $^{\circ}$ \\
\hline $22-23$ & 61.3 & & & & & & \\
\hline $22-24$ & 77.5 & $23-24$ & 89.8 & & & & \\
\hline $22-25$ & 37.9 & $23-25$ & 93.2 & $24-25$ & 97.3 & & \\
\hline $22-26$ & 69.13 & $23-26$ & 26.8 & $24-26$ & 65.5 & $25-26$ & 106.8 \\
\hline
\end{tabular}



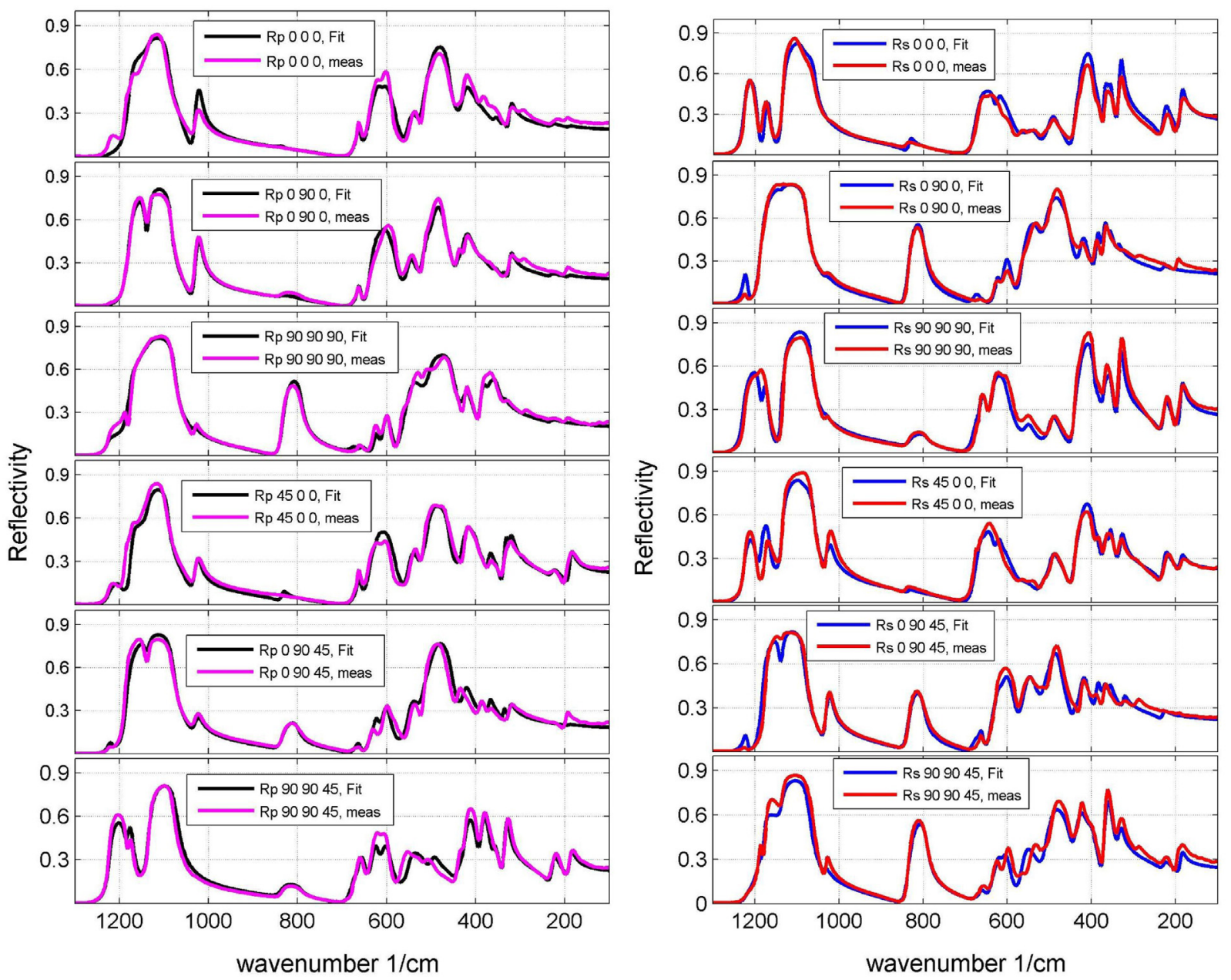

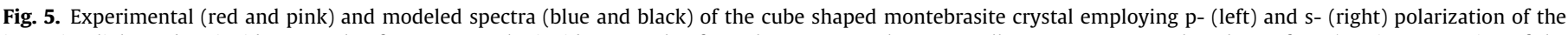

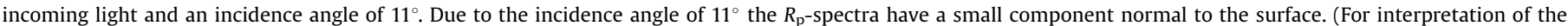
references to color in this figure legend, the reader is referred to the web version of this article.)

\subsection{Raman scattering results for montebrasite}

Polarized Raman spectra of the montebrasite crystal were recorded on the three planes, for parallel and perpendicularly polarized light. All the spectra show the same features, since triclinic Raman tensors have, in principle, non-null elements for

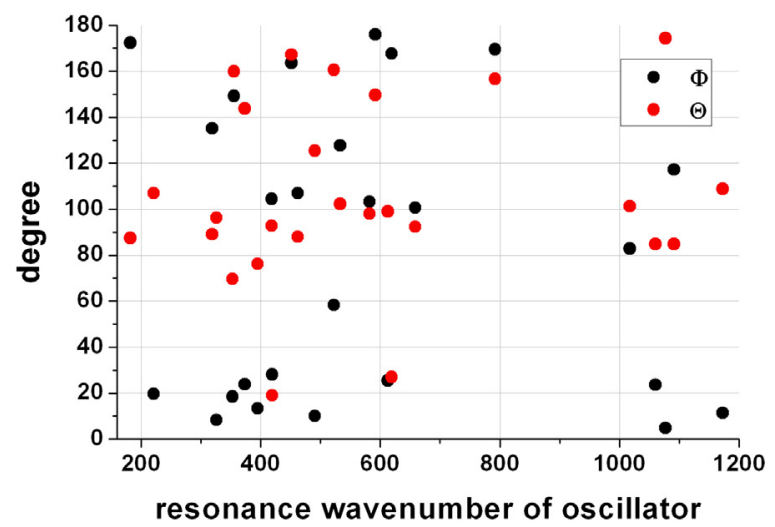

Fig. 6. Calculated orientation angles for the fitted oscillators. The orientation angles are given with respect to the face normal of the cube face of which the $R_{\mathrm{s}} / R_{\mathrm{p}} 000 / 45$ 00 spectra are taken. The angle distribution shows roughly three different directions of the transition moments, which indicates almost orthorhombic-like IR spectral behavior. any scattering configurations. However, the intensities of the individual bands depend on the particular measuring configuration, as it can be seen in Fig. 8, which shows the parallel-polarized Raman spectra of montebrasite taken on face 2, i.e., $a c^{*}$, with polarizers along $a$ or $c^{*}$ sample axes. We note particularly a high intensity of $\mathrm{OH}$ stretching band around $3380 \mathrm{~cm}^{-1}$ for $a$-polarization, and that some bands seem to vanish for one polarization, whereas they are intense for perpendicular polarization. It is important to mention that, as montebrasite belongs to a centrosymmetric group, the Raman phonon frequencies do not depend on the measuring configuration (indeed, we checked this point by comparing spectra taken onto different crystal faces and orientations, which give always the same positions for all bands). Once the intensity of the bands depends, however, on the polarization direction, we have recorded the Raman spectra for several polarization directions. Fig. 9 shows the Raman spectra recorded on the $a c^{*}$-plane for polarized light making an angle from 0 to $180^{\circ}$ with the $a$-direction $\left(90^{\circ}\right.$ means $c^{*}$ polarization, the sample was turned clockwise around the $b^{*}$-axis).

Fig. 9a $\left(100-580 \mathrm{~cm}^{-1}\right)$ shows the variations of the 14 lowestfrequency modes with polarization angle. These modes correspond roughly $\mathrm{Li}-\mathrm{O}$ vibration, $\mathrm{AlO}_{6}$ bending and $\mathrm{PO}_{4}$ bending modes [30], but a definite assignment was not done so far and it is beyond our purposes. Fig. $9 \mathrm{~b}\left(560-950 \mathrm{~cm}^{-1}\right)$ shows the behavior of three $\mathrm{AlO}_{6}$ stretching modes (\#15 to 17) and a hydroxyl-related mode at around $798 \mathrm{~cm}^{-1}$, likely due to $\mathrm{P}-\mathrm{OH}$ stretching and $\mathrm{P}-\mathrm{O}-\mathrm{H}$ 

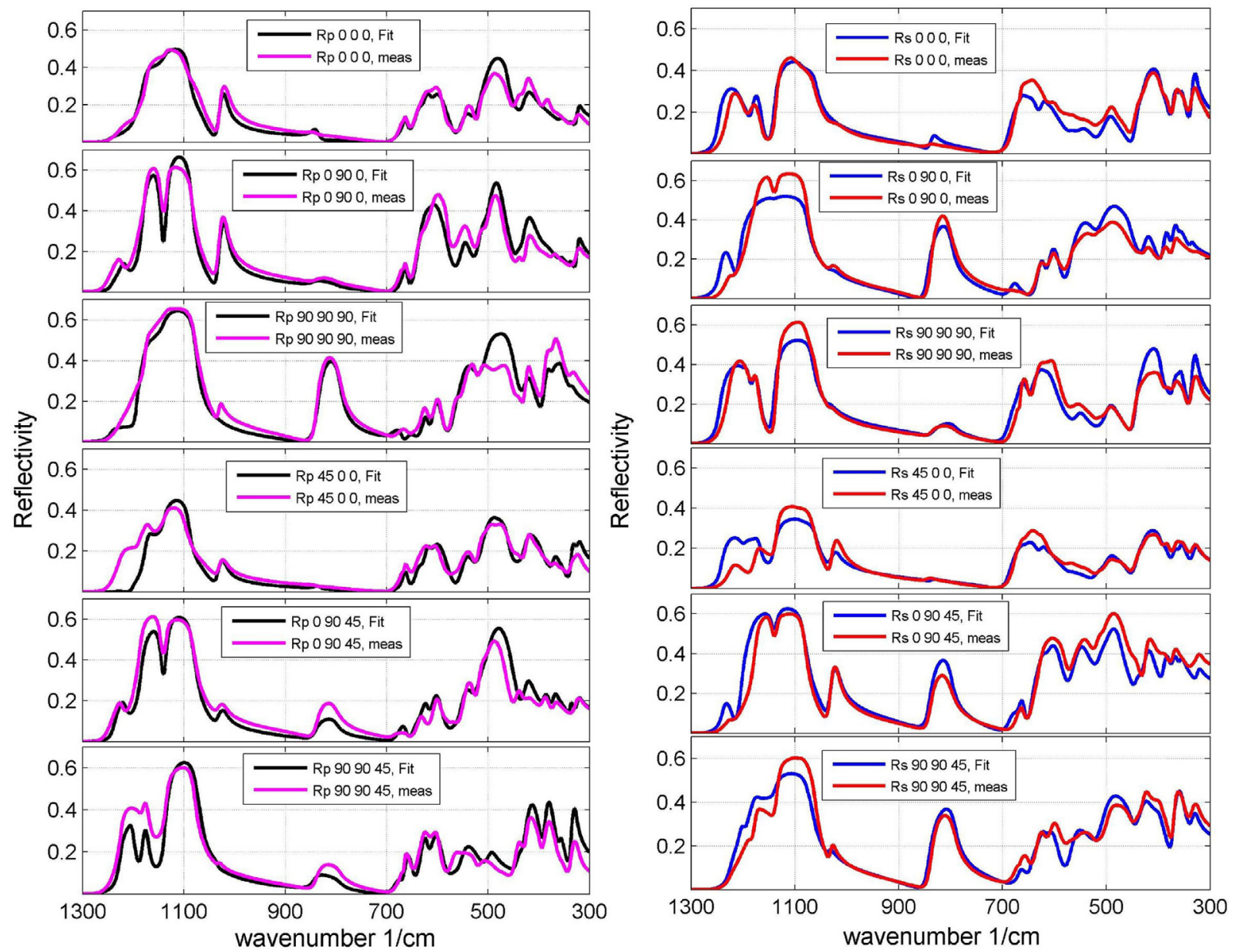

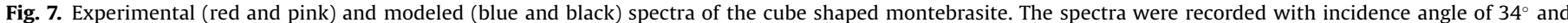

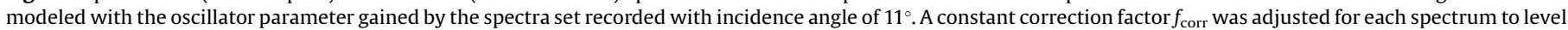

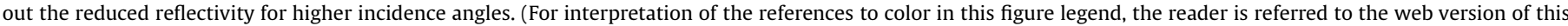
article.)

torsion [32,40], as observed in the IR spectra. Fig. 9c presents the variations of the four $\mathrm{PO}_{4}$ stretching modes (\#19 to 22) and the $\mathrm{OH}$ bending mode (\#23) with polarization angle. The spectra shown in Fig. 9a-c (along with the $\mathrm{OH}$ stretching mode at $3383 \mathrm{~cm}^{-1}$ ) allowed us to obtain the complete set of 24 Raman-active modes of montebrasite, presented in Table 4.

A weak feature at around $873 \mathrm{~cm}^{-1}$ (whose intensity is comparable with that of the $548 \mathrm{~cm}^{-1}$ band) was not considered in our analysis. We think that it could be a signature of anharmonicity, as are some other very weak features around $1581,2240,2360,3255$ and $3513 \mathrm{~cm}^{-1}$. The spectra of Fig. 9a-c also show that one must take care in analyzing Raman spectra of triclinic crystals, once some bands can be absent in some orientations, as exemplified in Fig. 9d that presents the intensity variations of four chosen bands. Notice that the intensity of bands around 277, 798 and $1187 \mathrm{~cm}^{-1}$ vanish in parallel light for polarizers along the $c^{*}$ direction (having maxima along the $a$ direction). Conversely, the $139 \mathrm{~cm}^{-1}$ band has its maximum along $c^{*}$ and vanish along $a$.

Now by taking the IR and Raman spectra altogether, we can conclude that the investigated montebrasite sample presented a very good crystallographic order. Indeed, we were able to depict all the well-behaved 27 IR and 24 Raman modes predicted by group theory for the $\bar{P} \overline{1}$ crystal structure. The Raman modes did not show any behavior of polar elliptical phonons, i.e., the IR modes were not activated in the Raman spectra and the frequencies were independent on the particular scattering configuration. Conversely, the IR modes showed normal behavior of polar phonons. As a consequence, we can conclude that defects play no significant role in this natural crystal, within the limits of our experimental

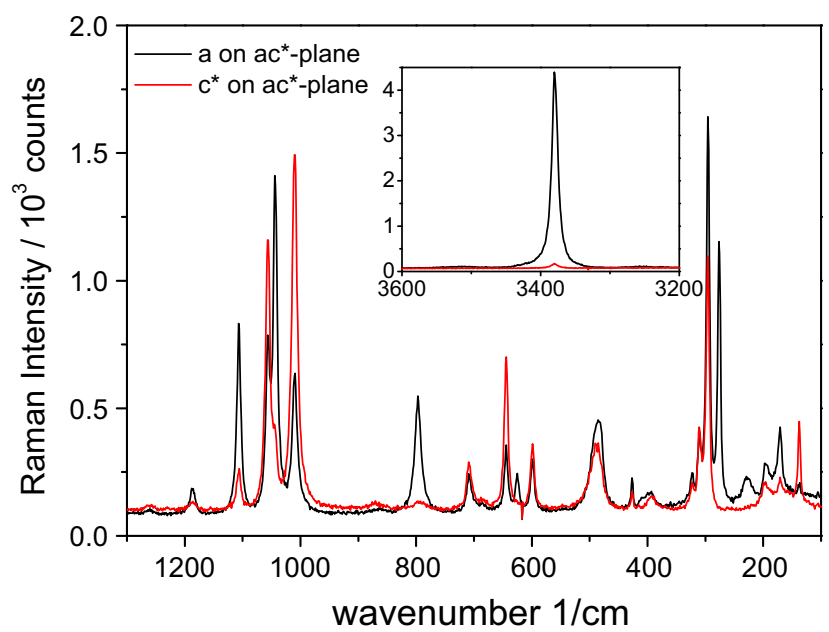

Fig. 8. Parallel-polarized Raman spectra of montebrasite on face $2\left(a c^{*}\right)$, along axes $a$ and $c^{*}$, from 100 to $1300 \mathrm{~cm}^{-1}$. The inset shows the spectral region around the $\mathrm{OH}$ stretching mode. 

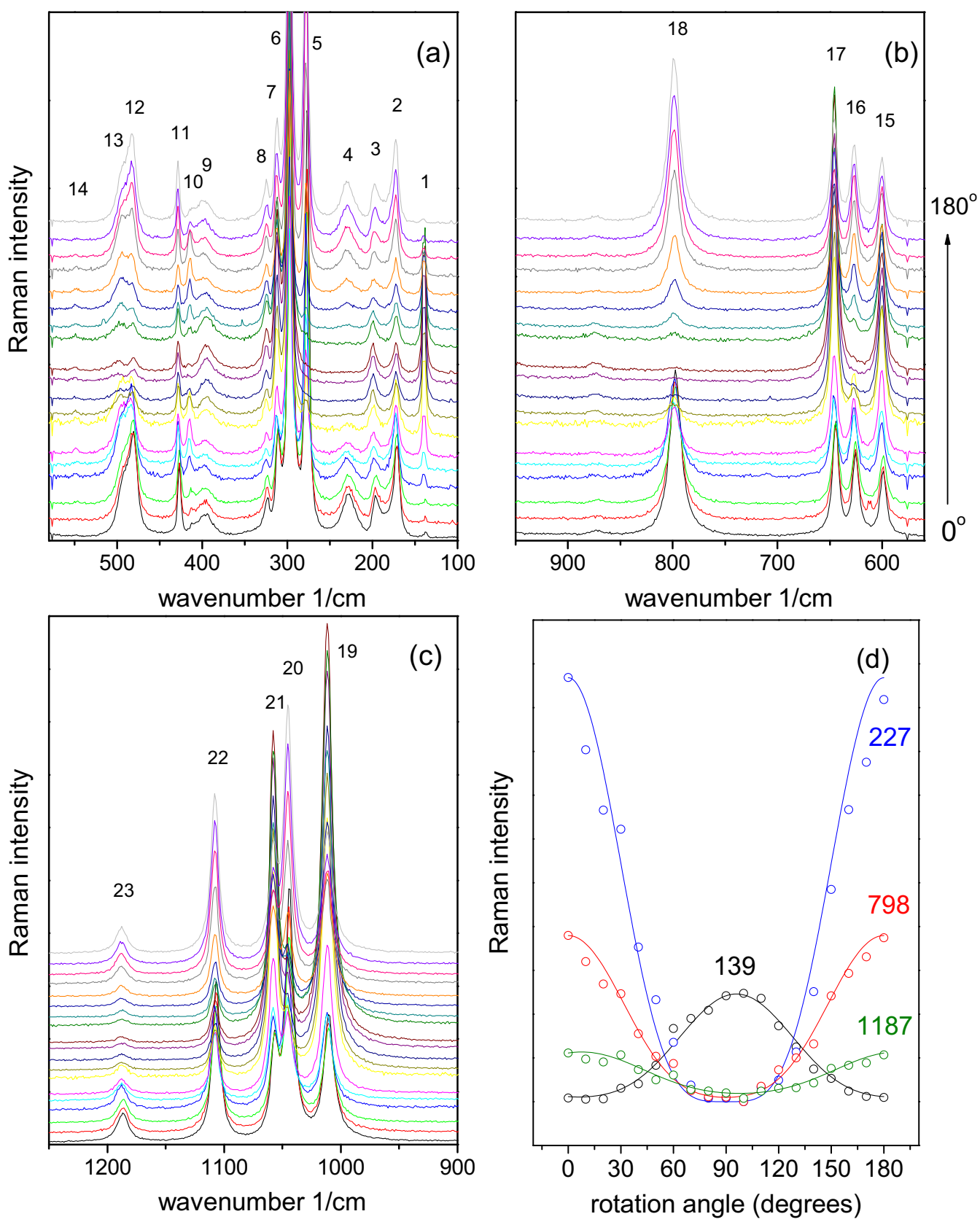

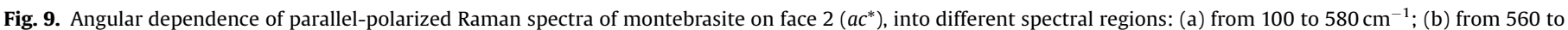

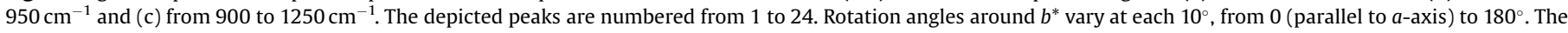
sample was rotated clockwise. (d) The intensity dependence of four chosen bands as a function of the rotation angle.

accuracy. Also, and with important consequences for the analysis of montebrasite system, our results are compatible with fully occupied atomic sites, as proposed by Baur [27]. In fact, halfoccupied or partially occupied lithium sites as proposed by other authors $[31,33,34]$ would lead to an increasing number of modes because vacancies must be counted (three additional IR and three new Raman modes not observed here). Moreover, because the Li vacancies in such structure would be disordered, this would lead to broad IR and Raman bands. As it can be seen in our spectra and fitting bands, the depicted IR and Raman modes are relatively narrow, which is not compatible with a disordered structure. Since montebrasite-amblygonite constitutes a natural system, our conclusion is that the analysis of our montebrasite sample showed that it has a well-ordered structure with just one fully occupied Li site.
Table 4

Depicted Raman modes for montebrasite.

\begin{tabular}{rlllll}
\hline \# & This work & Rondeau et al. [30] & \#Cont. & This work & Rondeau et al. [30] \\
\hline 1 & $139(5)$ & - & 13 & $493(11)$ & - \\
2 & $172(8)$ & 170 & 14 & $548(8)$ & \\
3 & $198(8)$ & - & 15 & $600(7)$ & 600 \\
4 & $229(16)$ & - & 16 & $626(7)$ & - \\
5 & $277(6)$ & - & 17 & $646(7)$ & 645 \\
6 & $297(4)$ & 295 & 18 & $798(13)$ & - \\
7 & $312(5)$ & - & 19 & $1012(11)$ & 1012 \\
8 & $324(5)$ & - & 20 & $1046(9)$ & 1045 \\
9 & $396(11)$ & - & 21 & $1057(8)$ & 1057 \\
10 & $415(6)$ & - & 22 & $1108(10)$ & 1107 \\
11 & $428(5)$ & 427 & 23 & $1187(11)$ & - \\
12 & $483(9)$ & - & 24 & $3383(11)$ & 3379 \\
\hline
\end{tabular}




\section{Conclusions}

We have performed dispersion analysis of a triclinic montebrasite crystal, from the polarized-reflectivity infrared spectra taken at low-incidence angle and checked for a higher incidence angle. The spectra revealed 27 well-behaved polar modes, in agreement with group theory for the $\mathrm{P} \overline{1}$ space group. The complete set of dispersion parameters was obtained, including the azimuth and co-elevation angles for each polar (ungerade) phonon. Polarized Raman spectra in several scattering configurations allowed us to depict the 24 gerade modes predicted for this structure. For these non-polar phonons, frequencies and bandwidths were independent of the scattering geometry, while their scattered intensities depend individually on the particular scattering geometry. The Raman and IR selection rules were well obeyed and all the observed bands were relatively narrow. These facts altogether (number of modes, selection rules and bandwidths) are compatible with a well-ordered material with fully occupied atomic sites.

\section{Acknowledgments}

The Brazilian authors thank the Conselho Nacional de Desenvolvimento Científico e Tecnológico (CNPq) and the Fundação de Amparo à Pesquisa do Estado de Minas Gerais (FAPEMIG) for financial support. The German authors thank the Deutsche Forschungsgemeinschaft for financing the investigations that led to the development of the dispersion analysis formalism of triclinic crystals employed in this work.

\section{References}

[1] M. Czerny, Z. Phys 65 (1930) 600-631.

[2] W.G. Spitzer, D.A. Kleinman, D. Walsh, Phys. Rev. 113 (1959) 127-132.

[3] W.G. Spitzer, D.A. Kleinman, Phys. Rev. 121 (1961) 1324-1335.

[4] F. Gervais, P. Echegut, Infrared studies of incommensurate systems, in: R. Blinc, A.P. Levanyuk (Eds.), Incommensurate Phases in Dielectrics - Fundamentals, Amsterdam, North Holland, 1986, pp. 337-364.

[5] M. Balkanski, Optical properties due to phonons, in: T.S. Moss, M. Balkanski (Eds.), Handbook on Semiconductors, Amsterdan, North-Holland, 1980, pp. 497-543.

[6] E.E. Koch, A. Otto, K.L. Kliewer, Chem. Phys. 3 (1974) 362-369.

[7] J. Herranz, J.M. Delgado, Spectrochim. Acta A 31 (1975) 1255-1263.
[8] V.F. Pavinich, M.V. Belousov, Opt. Spectrosc. 45 (1978) 881-883.

[9] J.R. Aronson, A.G. Emslie, E.V. Miseo, E.M. Smith, P.F. Strong, Appl. Opt. 22 (1983) 4093-4098.

[10] A.B. Kuz'menko, E.A. Tishchenko, V.G. Orlov, J. Phys.: Condens. Matter 8 (1996) 6199-6212.

[11] A.B. Kuz'menko, D. van der Marel, P.J.M. van Bentum, E.A. Tishchenko, C. Presura, A.A. Bush, Phys. Rev. B 63 (2001) 94303.

[12] A. Gössling, T. Möller, W.-D. Stein, P. Becker, L. Bohatý, M. Grüninger, Phys. Stat Sol. (b) 242 (2005) R85-R87.

[13] J.L. Ribeiro, L.G. Vieira, I. Tarroso Gomes, D. Isakov, E. de Matos Gomes, M Belsley, J. Phys.: Condens. Matter 19 (2007) 176255.

[14] V. Ivanovski, T.G. Mayerhöefer, J. Popp, Vib. Spectrosc. 44 (2007) 369-374.

[15] V. Ivanovski, T.G. Mayerhöefer, J. Popp, Spectrochim. Acta A 68 (2007) 632-638.

[16] V. Ivanovski, T.G. Mayerhöefer, J. Popp, V.M. Petruševski, Spectrochim. Acta A 69 (2008) 629-641.

[17] V. Ivanovski, T.G. Mayerhöefer, J. Popp, Vib. Spectrosc. 47 (2008) 91-98.

[18] V. Ta Phuoc, C. Sellier, E. Janod, C. Marin, Phys. Rev. B 77 (2008) 075123.

[19] V. Ivanovski, G. Ivanovski, Spectrochim. Acta A 75 (2010) 1452-1461.

[20] T.G. Mayerhöfer, S. Webber, J. Popp, Opt. Commun. 284 (2011) 719-723.

[21] T.G. Mayerhöfer, V. Ivanovski, J. Popp, Vib. Spectrosc. 63 (2012) 396-403.

[22] T. Möller, P. Becker, L. Bohatý, J. Hemberger, M. Grüninger, Phys. Rev. B 90 (2014) 155105.

[23] A.G. Emslie, J.R. Aronson, J. Opt. Soc. Am. 73 (1983) 916-919.

[24] J.R. Aronson, A.G. Emslie, P.F. Strong, Appl. Opt. 24 (1985) 1200-1203.

[25] S. Höfer, J. Popp, T.G. Mayerhöfer, Vib. Spectrosc. 67 (2013) 44-54.

[26] S. Höfer, J. Popp, T.G. Mayerhöfer, Vib. Spectrosc. 72 (2014) 111-118.

[27] W.H. Baur, Acta Crystallogr. 12 (1959) 988-994.

[28] L.N. Dias, M.V.B. Pinheiro, R.L. Moreira, K. Krambrock, K.J. Guedes, L.A.D. Menezes Filho, J. Karfunkel, J. Schnellrath, R. Scholz, Am. Mineral. 96 (2011) 42-52.

[29] I. Černá, P. Černý, R.B. Ferguson, Am. Mineral. 58 (1973) 291-301.

[30] B. Rondeau, E. Fritsch, P. Lefevre, M. Guiraud, A.-M. Fransolet, Y. Lulzac, Can. Mineral. 44 (2006) 1109-1117.

[31] L.A. Groat, B.C. Chakoumakos, D.H. Brouwer, C.M. Hoffman, C.A. Fyfe, H. Morell, A.J. Schultz, Am. Mineral. 88 (2003) 195-210.

[32] A.-M. Fransolet, P. Tarte, Am. Mineral. 62 (1977) 559-564.

[33] L.A. Groat, M. Raudsepp, F.C. Hawthorne, T. Scott Ercit, B.L. Sherriff, J.S. Hartman, Am. Mineral. 75 (1990) 992-1008.

[34] V.I. Simonov, N.V. Belov, Kristallografiya 3 (1958) 428-437.

[35] P. Yeh, Optical Waves in Layered Media, Wiley, New York, 1988.

[36] D.L. Rousseau, R.P. Bauman, S.P.S. Porto, J. Raman Spectrosc. 10 (1981) 253-290.

[37] H.H. Adler, Am. Mineral. 49 (1964) 1002-1015.

[38] R.L. Frost, J. Čejka, G.A. Ayoko, M. Weier, Spectrochim. Acta A 66 (2007) 979-984.

[39] C.C. Pye, W.W. Rudolph, J. Phys. Chem. A 107 (2003) 8746-8755.

[40] N. Maubec, A. Lahfid, C. Lerouge, G. Wille, K. Michel, Spectrochim. Acta A 96 (2012) 925-939.

[41] D.A. Rusakov, A.A. Filaretov, M.N. Bubentsova, V.P. Danilov, L.N. Komissarova, Russ. J. Inorg. Chem. 51 (2006) 852-861.

[42] D.S. Andrianov, A.S. Blagoveshchenskii, T.D. Kolomiitsova, D.N. Shchepkin, Opt. Spectrosc. 94 (2003) 513-527. 